\title{
Rare Cause of Pacemaker Lead Interference: Tricuspid Valve Myxoma
}

\author{
Agostino Pozzi ${ }^{* 1}$, Alessandra Sala ${ }^{2}$, Marco Bartolucci ${ }^{3}$ \\ ${ }^{1 *}$ Department of General Surgery, San Raffaele Hospital, Vita-Salute San Raffaele University, Milan, Italy. \\ ${ }^{2}$ Department of Cardiac Surgery, San Raffaele Hospital, Vita-Salute San Raffaele University, Milan, Italy. \\ ${ }^{3}$ San Raffaele Hospital, Vita-Salute San Raffaele University, Milan, Italy.
}

${ }^{*}$ Correspondence to: Agostino Pozzi, Department of General Surgery, San Raffaele Hospital, Vita-Salute San Raffaele University, Milan, Italy; Email: agostinopozzi@ hotmail.it

Received: September 15; Accepted: October 25, 2016; Published: November 11, 2016

\begin{abstract}
Introduction: Primary tumors of the heart are a rare finding, and the most common benign tumors are cardiac myxomas, accounting for up to $80 \%$ of cases. They are of endocardial origin therefore the prevalence of myxomas in the atrial septum is highest. Among myxomas left atrial ones are the most common $(75 \%)$, followed by right atrial ones $(18 \%)$.

Case Presentation: We present a case of a cardiac myxoma of the tricuspid valve with a very unusual clinical presentation of ventricular pacemaker leads interference. A 71-year-old male with history of chronic atrial fibrillation, acute coronary artery syndrome, heart failure with ICD-CRT(Implantable cardioverter Defibrillator-Cardiac Resynchronization Therapy) implantation and mitral regurgitation. A transthoracic echocardiogram showed a right atrial filamentous mass originating from the posterior leaflet of the tricuspid valve and causing inappropriate sensing of the ventricular pacemaker leads. He underwent resection of the mass which on pathology was revealed to be a cardiac myxoma.

Conclusions: Cardiac masses are relatively rare findings and the clinical presentation is characterized by nonspecific signs and symptoms, such as embolism, dyspnea, pulmonary edema, fever, fatigue and weight loss, which lead to a wide differential diagnosis. It is crucial for clinicians to consider cardiac myxomas among the possible diagnoses. This case report described an unusual clinical presentation of cardiac myxomas.
\end{abstract}

Key words: Cardiac myxoma, tricuspid valve myxoma, pacemaker leads

\section{Introduction}

Primary tumors of the heart are a rare finding, with an incidence of 0.0017 to $0.33 \%$ at autopsy [1]. The most common benign tumors are cardiac myxomas, accounting for up to $80 \%$ of cases ${ }^{2}$. Cardiac myxomas are defined as neoplasms composed of stellate to plump cytologically bland mesenchymal cells set in a myxoid stroma [2]. They are of endocardial origin, and project from the endocardium into the cardiac chambers; cells giving rise to such tumors are thought to be multipotent mesenchymal cells that persist as embryonal residues [3]. As a result, the prevalence of myxomas in the atrial septum is highest.

We are reporting a case of cardiac myxoma of the right side of the heart with an unusual initial presentation of pacemaker leads interference.

\section{Case Report}

A 71-year-old male with past medical history of chronic atrial fibrillation, acute coronary artery syndrome treated with multiple stents on the circumflex coronary artery, heart failure with ICD-CRT implantation and mitral regurgitation treated with the percutaneous MitraClip system, was presented to our department due to repeated episodes of noise sensed from the right ventricular electrode recognized inappropriately as ventricular fibrillation (no shock delivered due to the short-lasting episode).
The goals of the initial evaluation were to ascertain whether the documented episode could be reproduced and whether the ICD-CRT leads were correctly positioned within the right ventricle. A trial was done and upon deep breathing a long episode of noise interference was detected and inappropriately sensed as an $\mathrm{R}$ wave, with consequent pacing deficit and asystole, in a pacemaker-dependent patient.

Both chest x-ray (CXR) and transthoracic echocardiography (TTE) were later performed to assess the pacemaker catheters and cardiac function.

TTE showed good results of the previous MitraClip implant, with mild mitral regurgitation; dilated left atrium and left ventricle (DTD $60 \mathrm{~mm}, \mathrm{EF} 50 \%$ ); presence of a filiform mass attached to the atrial aspect of the tricuspid valve posterior leaflet of $12 \mathrm{~mm}$ in dimensions, consistent with a hypothesis of fibroelastoma; moderate tricuspid valve regurgitation $(++)$ and presence of pacemaker catheter within the right ventricle.

Laboratory findings were as follows: WBC $10.5 \times 10^{9} / \mathrm{L}, \mathrm{Hb} 13.8$ g/dL, Hct $41.2 \%$, Platelets $170 \times 10^{9} / \mathrm{L}$. Electrolyte panel showed: $\mathrm{Na}^{+}$ $139.2 \mathrm{mmol} / \mathrm{L}, \mathrm{K}^{+} 4.60 \mathrm{mmol} / \mathrm{L}, \mathrm{Mg}^{2+} 0.92 \mathrm{mmol} / \mathrm{L}$, Creatinine 1.23 $\mathrm{mg} / \mathrm{dL}$, indirect bilirubin $0.62 \mathrm{mg} / \mathrm{dL}, \mathrm{LDH} 210 \mathrm{U} / \mathrm{L}$, and glucose 97 $\mathrm{mg} / \mathrm{dL}$. 
A diagnosis of tricuspid valve fibroelastoma was made and the patient underwent surgery with resection of the right atrial mass (reported intraoperative dimensions of $15 \mathrm{~mm}$ ), reconstruction of the posterior leaflet of the tricuspid valve, tricuspid valve annuloplasty due to annular dilation resulting in tricuspid insufficiency, and closure of an atrial septal defect (ostium secundum type).

Postoperative EKG showed normal pacemaker-dependent rhythm, with good function and positioning of the ICD-CRT catheters (on chest $\mathrm{x}$-ray and TTE). The patient recovered well and was discharged on post-operative day 4 . Pre-discharge transthoracic echocardiography showed good result of mass excision, no residual tricuspid valve regurgitation or stenosis, and a left ventricular ejection fraction of $50 \%$.

Unexpectedly, tissue biopsy of the mass came back positive for cardiac myxoma.

\section{Discussion}

Cardiac myxomas are rare benign tumors accounting for $45 \%$ of primary cardiac tumors in adults, and among myxomas left atrial ones are the most common (75\%), followed by right atrial (18\%), left and right ventricular masses (2.5-4\%) [4].

Two types of macroscopic appearance are observed: polypoid and papillary types [5]. The former is the most common, usually compact, round or oval with a smooth or gently lobulated surface. The less common papillary myxomas have a surface with multiple fine villous extensions; these tend to be gelatinous and fragile and are at increased risk of breaking off [5]. The rate of growth of myxomas is unknown, however they are thought to grow rather quickly [6].

Myxomas can be detected in any age group but are particularly frequent between the third and sixth decade, and mainly in females [7]. Most commonly occurring sporadically, familial cardiac myxomas have been reported as part of a the Carney complex syndrome; a disorder of young (mean age 24 years) men (66\%), often multicentric and associated with other rare conditions, such as skin myxomas, skin pigmented lesions and endocrine tumors [8].

\section{Clinical Characteristics}

Clinical features of myxomas are determined by their location, size and mobility. They may be completely asymptomatic, especially in the case of small masses ( $20 \%$ of cases). However, the most common triad of presentation is embolism, intracardiac obstruction and constitutional symptoms, such as fatigue, fever, exanthematous rash, myalgia, weight loss and laboratory abnormalities. Embolism occurs in approximately 30 to $40 \%$ of patients, and being the majority located in the left atrium, systemic embolism to cerebral arteries is particularly frequent [9].

Myxomas give rise to signs of obstructed filling of the left and right ventricles with subsequent dyspnea, pulmonary edema and heart failure. Furthermore, the rocking back and forth of the mass on the atrioventricular valves may be responsible for damage to the leaflets or to the subvalvular apparatus, resulting in chordal rupture and valve insufficiency [5].

\section{Imaging techniques}

Among the various diagnostic tests available, echocardiography, computed tomography (CT) scan and magnetic resonance imaging (MRI) are of primary importance for the detection of cardiac masses.

Echocardiography is the gold standard, readily available and non-invasive tool. Both transthoracic and transesophageal echocardiography are used to determine location, size and shape; however, the latter is particularly useful in detecting the site of insertion and morphologic features, such as cysts and calcification [10]. However, the technique is operator-dependent and lacks the ability of discriminating specific tissue qualities.

Cardiac tumors can be identified by CT and MRI; both have emerged as alternative techniques, non-invasive, operator independent and capable of providing sectional and orthogonal views. MRI is capable of demonstrating tissue characteristics therefore can demonstrate masses of various etiologies [11]. As a consequence, MRI is better suited for suggesting etiology, delineating the extent, the relationship to adjacent structures and presence of any hemodynamic effects, despite the possible limitations related to cardiac and respiratory motion artifacts [12]. CT scan is another important, noninvasive tool with a higher density resolution to distinguish soft tissue mass and measure both fatty content and calcifications. This allows not only measurement of the morphologic character of cardiac myxomas (solid, liquid, hemorrhagic, fatty) but also the tumor pedicle diameter and its modification over time [13].

\section{Differential diagnosis}

Whenever an intracardiac mass is detected, differential diagnosis takes into account benign and malignant primary heart tumors, metastasis, thrombi and vegetations. Secondary or metastatic tumors, via lymphatic or hematogenous spread, are 20-40 times more frequent than primary cardiac tumors. Both are accompanied by constitutional symptoms, like fever, anemia, weight loss, leukocytosis and elevated erythrocyte sedimentation rate (ESR) values [14].

The formation of thrombi, instead, mainly occurs in patients with regional or global wall-motion abnormalities, like dilated cardiomyopathy, myocardial infarction and atrial fibrillation. Left atrial thrombi are generally attached to the posterior left atrial wall, while ventricular thrombi are rare in patients with normal left ventricular function [15]. Vegetations are another important aspect to be evaluated and ruled out.

\section{Treatment}

Treatment of choice is surgical excision performed promptly to avoid embolic complications. The root of the pedicle should be excised and in the case of atrial septal defect it should be corrected via direct suture or pericardial patch closure. Reported short and long-term prognosis is generally very good, with an operative mortality as low as $0-3 \%$ [16]. Postoperative atrial arrhythmias or atrioventricular conduction abnormalities have been described in the literature [17]. Moreover, patients may have increased risk of developing recurrence of myxomas or other cardiac masses, as high as $5 \%$, posing indication to thorough follow-up [18]. 


\section{Conclusion}

Cardiac masses are relatively rare findings and the clinical presentation is characterized by nonspecific signs and symptoms which lead to a wide differential diagnosis. However, it is critical for clinicians to consider cardiac myxomas, the most common benign cardiac mass, as part of the possible diagnoses. This is a rare case of ventricular pacemaker lead interference by a tricuspid valve myxoma.

\section{Disclosures}

Authors have no conflicts of interest or financial ties to disclose.

\section{References}

1. Wold LE, Lie JT (1980) Cardiac myxomas: a clinicopathologic profile. Am J Pathol 101: 219-240. [crossref]

2. Travis WD, Brambilla E, Müller-hermelink HK, Harris CC (2004) World Health Organization Classification of Tumours WHO Classification Tumours of the Lung , Pleura, Thymus and Heart Pathology \& Genetics Tumours of the Lung, Pleura, Thymus and Heart.

3. Vito A Di, Mignogna C, Donato G, A DV, Mignogna C, Donato G, Vito A Di (2015) The mysterious pathways of cardiac myxomas?: a review of histogenesis pathogenesis and pathology. Histopathology. 66:321-332.

4. MacGowan SW1, Sidhu P, Aherne T, Luke D, Wood AE, et al. (1993) Atrial myxoma: national incidence, diagnosis and surgical management. Ir J Med Sci 162: 223-226. [crossref]

5. Reynen K1 (1995) Cardiac myxomas. N Engl J Med 333: 1610-1617. [crossref]

6. Malekzadeh S1, Roberts WC (1989) Growth rate of left atrial myxoma. Am J Cardiol 64: 1075-1076. [crossref]

7. Wang JG, Li YJ, Liu H, Li NN, Zhao J, et al. (2012) Clinicopathologic analysis of cardiac myxomas: Seven years' experience with 61 patients. J Thorac Dis 4: 272-283. [crossref]

8. Carney JA (1985) Differences between nonfamilial and familial cardiac myxoma. Am J Surg Pathol 9: 53-55. [crossref]

9. Blondeau P1 (1990) Primary cardiac tumors--French studies of 533 cases. Thorac Cardiovasc Surg 38 Suppl 2: 192-195. [crossref]

10. Engberding R, Daniel W, Erbel R, Kasper W, Lestuzzi C, et al. (1993) Diagnosis of heart tumours by transesophageal echocardiography: a multicentre study in 154 patients. Eur Heart $J$ 14:1223-8.

11. Kaminaga T, Takeshita T, Kimura I (2003) Role of magnetic resonance imaging for evaluation of tumors in the cardiac region. Eur Radiol 4:L1-10.

12. Gulati G, Sharma S, Kothari SS, Juneja R, Saxena A, et al. (2004) Comparison of echo and MRI in the imaging evaluation of intracardiac masses. Cardiovasc Intervent Radiol 27: 459-469. [crossref]

13. Quan H, Liang P, Tan Y. The Value of Multi-slice CT Imaging in Cardiac Myxomas in Comparison with Follow-Up Screening in Thoracoscopic Surgery. Cell Biochem Biophys. 2015;73:565-569.

14. Chan O, Igwe M, Breburda CS, Amar S (2016) Burkitt Lymphoma Presenting as an Intracardiac Mass: Case Report and Review of Literature. Am J Case Rep 17: 553-558. [crossref]

15. Lee WC, Huang MP, Fu M (2015) Multiple intracardiac masses: myxoma, thrombus or metastasis: a case report. J Med Case Rep 9: 179. [crossref]

16. Rahmanian PB, Castillo JG, Sanz J, Adams DH, Filsoufi F (2010) Cardiac myxoma?: preoperative diagnosis using a multimodal imaging approach and surgical outcome in a large contemporary series. Interact Cardiovasc Thorac Surg 6:479-483.

17. Pinede L, Duhaut P, Loire R (2011) Clinical presentation of left atrial cardiac myxoma. A series of 112 consecutive cases. Medicine (Baltimore) 80:159-72.

18. Vohra HA, Vohra H, Patel RL (2002) Cardiac myxoma with three recurrences. $J R$ Soc Med 95: 252-253. [crossref]

Citation:

Agostino Pozzi, Alessandra Sala, Marco Bartolucci (2016) Rare Cause of Pacemaker Lead Interference: Tricuspid Valve Myxoma. Cancer Stud Ther J Volume 1(2): 1-3 